\title{
Voluntary exercise: Effects on ethanol-induced sleep in the C57BL/6J mouse
}

\author{
SANDRA MOLLENAUER, REBECCA BRYSON, and CHRISTINE PHILLIPS \\ San Diego State University, San Diego, California
}

(Robert M. Yaremko, Sponsor)

\begin{abstract}
After 5 weeks of voluntary wheel running, C57BL/6J mice were significantly resistant to the sleep-inducing effects of ethanol. Sixty-four mice, 32 males and 32 females, were assigned to wheel (free access to a running wheel in the home cage) or no-wheel conditions. At the end of the training period, the animals were removed from the exercise cages and tested for sensitivity to ethanol $(3.16 \mathrm{~g} / \mathrm{kg}, 20 \% \mathrm{w} / \mathrm{v})$, assessed by loss-of-righting reflex (LORR). Exercised animals showed significantly longer latency to LORR (fall time) and shorter duration of LORR (sleep time). Exercise caused a significant decrease in body weight in male, but not female, mice. The present results suggest that exercise training may be effective in reducing ethanol-induced sleep.
\end{abstract}

Until very recently, there has been little research on the possible effects of exercise training on response to drugs, including ethanol (Baak, 1990). In one of the few investigations in this area, Ardies, Morris, Erickson, and Farrar (1989) studied the effects of acute and chronic treadmill running in rats. They reported that a 60 -min session of forced treadmill running caused more rapid clearance of blood ethanol and that 7 weeks of exercise training enhanced this effect. Their work suggests that exercise training may help offset unwanted drug effects, such as ethanol-induced sleep.

In the present research, we explored the effects of voluntary wheel running on ethanol-induced sleep in the C57BL/6J mouse. The C57BL/6J is an inbred strain that has been widely used in alcohol research. Thus, previous research has established its normal ethanol sensitivity, as reflected in the loss-of-righting reflex (LORR) following a high dose of ethanol (Baker, A. Smolen, T. N. Smolen, Deitrich, 1987; A. Smolen, T. N. Smolen, \& Kamp, 1987). Previous research has also established that C57BL/6J mice show high levels of voluntary wheel running and maintain normal body weight during several months of running (Goodrick, 1978; Samorajski et al., 1985). In preliminary work monitoring number of wheel revolutions by computer, we obtained levels of activity comparable to previous reports and confirmed that C57BL/6J mice showed highly reliable activity levels, averaging approximately 500 revolutions per hour by the fifth week of exercise training. In the present experiment, animals were given 5 weeks of voluntary wheel running and then were compared with nonexercised controls for

Correspondence should be addressed to Sandra Mollenauer, Department of Psychology, San Diego State University, San Diego, California 92182-0350. ethanol sensitivity, assessed by the latency and duration of LORR after injection of $3.16 \mathrm{~g} / \mathrm{kg}$ of ethanol.

\section{METHOD}

\section{Animals}

The animals were 64 C57BL/6J mice, 2 months old at the beginning of the experiment. Four animals were lost during the course of the experiment, 3 as a consequence of apparatus failure and 1 as a consequence of the ethanol injection. All animals were third-generation offspring bred from stock obtained from Jackson Laboratories (Bar Harbor, ME). The animals were separated by sex at approximately 1 month of age and were reared in same-sex litter groups, with no more than 4 animals per cage. They were maintained on a 12-h light:dark cycle, adjusted so that all sleep tests could be conducted during the active phase of the animals' cycle. The light phase began at 1:00 p.m., and all injections were administered between 8:00 and 10:00 a.m., with exercise and nonexercise animals evenly represented across hours. The animals had ad-lib access to water and mouse lab chow throughout the course of the experiment and until $30 \mathrm{~min}$ before ethanol injections.

\section{Exercise Conditions}

Beginning at 2 months of age, equal numbers of male and female mice were randomly assigned to wheel or no-wheel conditions, with body weight balanced across conditions. Each animal was individually housed in a rectangular acrylic home cage, $25 \times 45 \mathrm{~cm}$ and $25 \mathrm{~cm}$ deep, with a wire cloth floor and wire cloth lid. Half the cages were equipped with small rodent running wheels (Ward Co.), measuring $18 \mathrm{~cm}$ in diameter. The cages were housed in large sound-attenuated chambers, equipped with fans and low-watt lighting to maintain the light:dark cycle. Each chamber held four wheel and four nonwheel cages, with males and females housed in separate chambers. Chambers were opened for servicing at 12:00 p.m. each day, and the animals were not otherwise disturbed. The experiment was conducted in two complete replications.

\section{Procedure}

After 5 weeks in wheel or no-wheel conditions, the animals were tested for ethanol sensitivity, assessed by LORR, following an intraperitoneal injection of $3.16 \mathrm{~g} / \mathrm{kg}$ ethanol (20\% w/v in normal saline). This dose was established in pilot work with animals maintained and tested on the same schedule. Each animal was removed from its chamber $30 \mathrm{~min}$ before injection and placed in an individual holding cage. After injection, the animal was returned to the holding cage; at the first sign of LORR, it was placed on its back in a V-shaped trough. Fall time, or latency 
to loss of the reflex, was recorded when the animal could not right itself three times within a 30-sec period; the same criterion was used to define sleep time, or the duration of LORR.

\section{RESULTS}

The effects of voluntary wheel running on LORR and body weight are summarized in Figure 1. The data for each measure were analyzed by analysis of variance with three between factors: wheel, gender, and replication. Fall time (i.e., time to LORR) is presented in the upper panel of the figure. As the figure suggests, the animals that had wheels took significantly longer to fall $[F(1,52)=4.04$, $p<.05$ ]. The main effect of gender was not significant $(F<1)$, nor was the interaction between wheel and
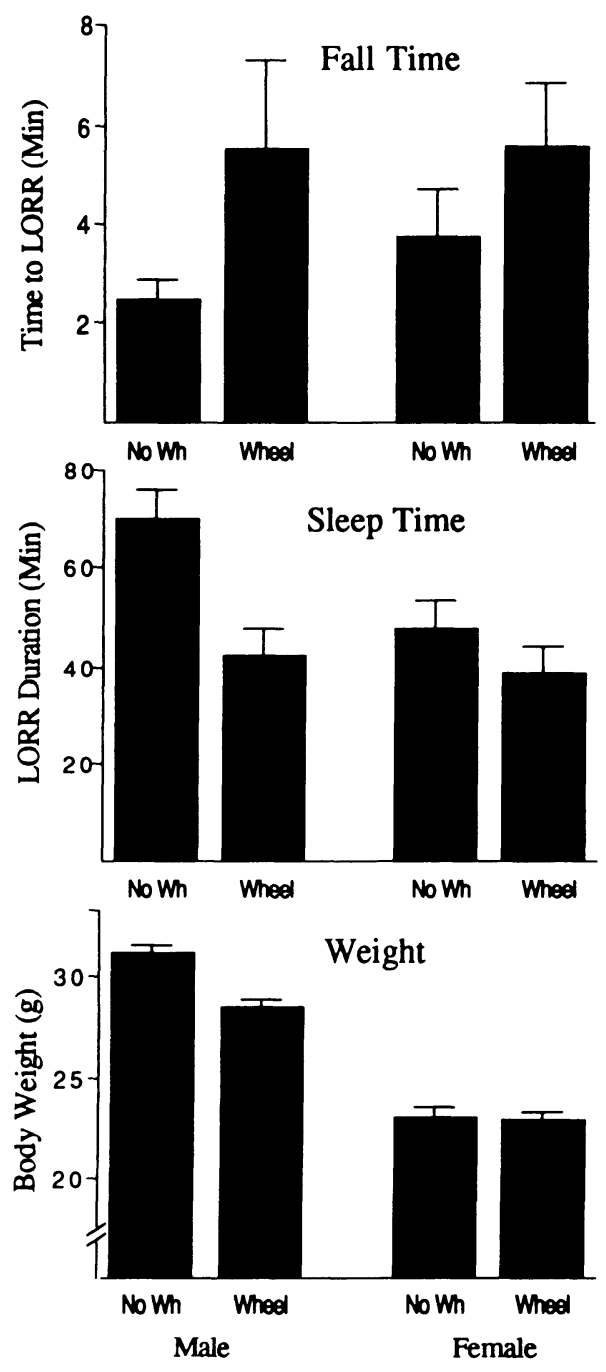

Figure 1. Upper panel: mean latency to LORR (fall time), $\pm S E M$, after $3.16 \mathrm{~g} / \mathrm{kg}(20 \% \mathrm{w} / \mathrm{v})$ ethanol. Middle panel: mean duration of LORR (sleep time), $\pm S E M$, after $3.16 \mathrm{~g} / \mathrm{kg}(20 \% \mathrm{w} / \mathrm{v})$ ethanol. Lower panel: mean body weight (in grams), $\pm S E M$, for male and female mice, after 5 weeks of voluntary exercise (Wheel) or no exercise (No Wh). gender $(F<1)$. The factor of replication was not significant as a main effect, nor did it interact with either of the other factors.

Sleep time (i.e., duration of LORR) is presented in the middle panel of Figure 1. As the figure shows, the animals that had wheels regained the righting reflex significantly faster, or, in other words, showed significantly less ethanol-induced sleep $[F(1,52)=14.03, p<.01]$. There was also a significant effect of gender, in which the males showed longer sleep than did the females $[F(1,52)=6.81$, $p<.05]$, but the interaction between wheel and gender was not significant $[F(1,52)=3.21]$. The main effect of replication was significant $[F(1,52)=13.26, p<.01]$; however, most important for the interpretation of these results, replication did not interact with either of the other variables.

Effects of exercise on body weight are presented in the lower panel of Figure 1. As the figure shows, the male mice were significantly heavier than were the female mice $[F(1,52)=257.6, p<.01]$, and there was a significant interaction between wheel and gender $[F(1,52)=7.87$, $p<.01]$, in which wheel running resulted in a significant reduction in body weight for the males $[F(1,29)=$ $21.22, p<.01]$, but not for the females $(F<1)$. The factor of replication was also significant $[F(1,52)=6.78$, $p<.05$ ]; again, however, most important for interpreting these results, replication did not interact significantly with either of the other factors.

\section{DISCUSSION}

After 5 weeks of voluntary exercise, the C57BL/6J mice were significantly resistant to the sleep-inducing effects of ethanol (Figure 1). The mice that had access to running wheels took significantly longer to lose the righting reflex after an injection of $3.16 \mathrm{~g} / \mathrm{kg}$ of ethanol and also regained the righting reflex significantly faster than did the nonexercised controls. An important feature of the present research is the fact that the animals were not returned to the exercise chambers after ethanol injection, but instead were placed in a holding cage until LORR occurred. Thus, the present results provide evidence of exercise training on subsequent ethanol response.

A number of physiological factors may have contributed to the present results. Duration of LORR is thought to reflect the animal's sensitivity to the anesthetic effects of ethanol (A. Smolen \& T. N. Smolen, 1987). In this view, the animal awakens when blood and, therefore, brain ethanol reaches the threshold level characteristic of the breed. Thus, any factor that altered the distribution of ethanol to blood and brain could be expected to affect sleep time. In the present research, voluntary wheel running may have altered ethanol distribution by causing changes in body composition. The analysis of weight data showed that access to running wheels resulted in small but reliable differences in body weight for the male, but not the female, mice. These weight differences could not account for the present results on the basis of dosage volume alone. However, it could be that the exercised animals had different body compositions, with proportionately less body fat and more body water. Faulkner, Cantleberry, Watts, and Hussain (1990) demonstrated that body composition is especially critical in interpreting effects of ethanol, which is not distributed to body fat. In the present research, the exercised animals may have had proportionately more body water and, thus, a higher volume of distribution for ethanol, or an effectively lower dose. Exercise has, in fact, been shown to alter the volume of distribution of a number of drugs (Baak, 1990).

The present results are also compatible with the idea that exercise causes accelerated clearance of ethanol. Intense exercise imposed after ethanol administration has recently been shown to cause an increased 
rate of ethanol clearance (Ardies et al., 1989; Schurch, Radimsky, Iffland, \& Hollmann, 1982). Ardies et al. also reported that 7 weeks of exercise training caused a significant increase in the activity of the hepatic microsomal ethanol oxidizing system (MEOS) of trained rats, tested at rest. An earlier study of hexobarbital sleep times in rats also suggested that chronic exercise caused acceleration of hepatic MEOS activity (Frenkl, Gyore, \& Szeberenyi, 1980). Thus, one possible explanation of the present results is that voluntary wheel running caused an alteration in hepatic activity that, in turn, caused more rapid clearance of ethanol. To the extent that prior wheel running caused an attenuation of ethanol-induced hypothermia, this, too, would be expected to accelerate ethanol clearance (Romm \& Collins, 1987). We are currently exploring the effects of voluntary wheel running on ethanol clearance rates and body temperature.

\section{REFERENCES}

Ardies, C. M., Morris, G. S., Erickson, C. K., \& FArrar, R. P. (1989). Both acute and chronic exercise enhance in vivo ethanol clearance in rats. Journal of Applied Physiology, 66, 555-560.

BAAK, VAN M. A. (1990). Influence of exercise on the pharmacokinetics of drugs. Clinical Pharmacokinetics, 19, 32-43.

Baker, R. C., Smolen, A., Smolen, T. N., Deitrich, R. A. (1987). Relationship between acute ethanol-related responses in Long-Sleep and Short-Sleep mice. Alcoholism: Clinical \& Experimental Research, 11, 574-578.

Faulkner, T. P., Cantleberry, S. B., Watts, V. J., \& Hussain,
A. S. (1990). Comparative pharmacokinetics of ethanol in inbred strains of mice using doses based on total body water. Alcoholism: Clinical \& Experimental Research, 14, 82-86.

FrenkL, R., GyORE, A., SZeberenyi, S. (1980). The effect of muscular exercise on the microsomal enzyme system of the rat liver. European Journal of Applied Physiology, 44, 135-140.

GooDRICK, C. L. (1978). Effect of voluntary wheel exercise on food intake, water intake, and body weight for C57BL/6J mice and mutations which differ in maximal body weight. Physiology \& Behavior, 21, 345-351.

Romm, E., \& Collins, A. C. (1987). Body temperature influences on ethanol elimination rate. Alcohol, 4, 189-198.

Samorajski, T., Delaney, C., Durham, L., Ordy, J. M., Johnson, J. A., DUNLAP, W. P. (1985). Effect of exercise on longevity, body weight, locomotor performance, and passive-avoidance memory of C57BL/6J mice. Neurobiology of Aging, 6, 17-24.

SChURCh, P. M., RADIMSKy, J., IFfland, R., \& HollmanN, W. (1982). The influence of moderate prolonged exercise and a low carbohydrate diet on ethanol elimination and on metabolism. European Journal of Applied Physiology, 48, 407-414.

SMOLEN, A., SMOLeN, T. N. (1987). Demonstration of a threshold concentration for ethanol at the time of regaining the righting response in Long-Sleep and Short-Sleep mice. Alcohol \& Drug Research, 7, 279-283.

Smolen, A., Smolen, T. N., \& KAmp, van de J. L. (1987). Sensitivity of inbred and selectively bred mice to ethanol. Alcohol, 4, 57-62.

(Manuscript received November 17, 1990.) 